\title{
Muco-adhesive Buccal Tablet Dosage Form
}

National Cancer Institute

\section{Source}

National Cancer Institute. Muco-adhesive Buccal Tablet Dosage Form. NCI Thesaurus.

Code C149670.

Solid single-dose preparation to be applied to the buccal mucosa to obtain a systemic delivery over an extended period of time. Mucoadhesive buccal tablets are usually prepared by compression of mixtures of powders or granulations into tablets with a shape suited for the intended use. They usually contain hydrophilic polymers, which on wetting with saliva produce a flexible hydrogel that adheres to the buccal mucosa. 